\title{
Sensory Memory for Odors Is Encoded in Spontaneous Correlated Activity Between Olfactory Glomeruli
}

\author{
Roberto F. Galán \\ galan@cnbc.cmu.edu \\ Institute for Theoretical Biology, Humboldt-Universität zu Berlin, 10115 Berlin, \\ Germany, and Department of Biological Sciences and Center for the Neural Basis of \\ Cognition, Mellon Institute, Carnegie Mellon University, Pittsburgh 15213, PA, \\ U.S.A.
}

Marcel Weidert

weidert@neurobiologie.fu-berlin.de

Randolf Menzel

menzel@neurobiologie.fu-berlin.de

Institute for Neurobiology, Freie Universität Berlin, 14195 Berlin, Germany

Andreas V. M. Herz

a.herz@biologie.hu-berlin.de

Institute for Theoretical Biology, Humboldt-Universität zu Berlin, 10115 Berlin,

Germany

C. Giovanni Galizia

galizia@ucr.edu

Department of Entomology, University of California, Riverside, CA 92521, U.S.A.

Sensory memory is a short-lived persistence of a sensory stimulus in the nervous system, such as iconic memory in the visual system. However, little is known about the mechanisms underlying olfactory sensory memory. We have therefore analyzed the effect of odor stimuli on the first odor-processing network in the honeybee brain, the antennal lobe, which corresponds to the vertebrate olfactory bulb. We stained output neurons with a calcium-sensitive dye and measured across-glomerular patterns of spontaneous activity before and after a stimulus. Such a single-odor presentation changed the relative timing of spontaneous activity across glomeruli in accordance with Hebb's theory of learning. Moreover, during the first few minutes after odor presentation, correlations between the spontaneous activity fluctuations suffice to reconstruct the stimulus. As spontaneous activity is ubiquitous in the brain, modifiable fluctuations could provide an ideal substrate for Hebbian reverberations and sensory memory in other neural systems. 


\section{Introduction}

Animals are able to evaluate a sensory stimulus for a certain time after stimulus offset, as in trace conditioning or delay learning (Clark, Manns, \& Squire, 2002; Grossmann, 1971). Such sensory memories have been extensively investigated in the visual system, such as for afterimages (i.e., visual persistence) or iconic memory and for the acoustic system (Crowder, 2003) and imply that a neural representation of the stimulus remains active after the stimulus, for example, during the time interval between a presented cue and a task to be performed or an association to be made. "Delayed matching and nonmatching to sample" paradigms also rely on a temporary storage of sensory information, generally referred to as working memory (Del Giudice, Fusi, \& Mattia, 2003). Such tasks have also been successfully solved by the honeybee, Apis mellifera, and prove that it possesses an exquisite sensory and working memory (Giurfa, Zhang, Jenett, Menzel, \& Srinivasan, 2001; Grossmann, 1971; Menzel, 2001).

Analyzing the neural basis of working memory in vertebrates, cortical neurons have been found that elevate their discharge rate during a delay period (Fuster \& Alexander, 1971). These findings suggest a straightforward realization of Hebbian "reverberations" (Hebb, 1949) in that persistently active delay cells provide the memory trace and thus allow the animal to compare sequentially presented stimuli (Amit \& Mongillo, 2003). In all of these studies, however, the investigation of sensory memory was embedded into a more complex paradigm in order to allow for a behavioral readout. Therefore, it cannot be excluded that the physiological traces of sensory memory contained a context-dependent or task-dependent component that is difficult to isolate. Taking a purely physiological approach and using the honeybee as an experimental animal, we asked whether a stimulus alone could modify brain activity in a way that would suggest a sensory memory. Odor stimuli are particularly salient for honeybees (Menzel \& Müller, 1996). We therefore sought an initial memory trace following a nonreinforced odor stimulus.

We find that the relative timing of spontaneous activity events in the antennal lobe is modified after a passive olfactory experience. This change follows the Hebbian covariance rule: pairs of coactivated or coinhibited glomeruli increased their correlation; glomeruli with an opposite response sign decreased their correlation (Sejnowski, 1977). Unlike the implications of Hebb's rules in developmental studies ("fire together, wire together"), the effect observed here was short-lived, with a decay time of a few minutes. We therefore suggest that this form of short-term Hebbian plasticity in the honeybee antennal lobe serves as an olfactory sensory memory.

\section{Materials and Methods}

2.1 Imaging. Bees were prepared as described elsewhere (Sachse \& Galizia, 2002). Briefly, forager bees (Apis mellifera carnica) were collected 
from the hive, cooled for anesthesia, and restrained in Plexiglas stages. A window was cut into the cuticle to expose the brain. Then the calciumsensitive tracer dye FURA-dextran (MW 10,000, Molecular Probes) was injected into the tract of PN axons that leads from the antennal lobe (AL) to the mushroom bodies (lateral antenno-cerebralis-tract, 1ACT). After 3 to 5 hours, the bees were tested for successful staining of PNs using a fluorescent microscope: successful staining was evident when the PN cell bodies were brightly fluorescent under $380 \mathrm{~nm}$ light exposure. We then recorded the calcium activity in the antennal lobe for 4 minutes, at a rate of six image pairs (340 nm/380 nm) per second. (Some animals were recorded for longer periods.) After 2 minutes (at half time), we presented an odor for 4 seconds, using a computer-controlled olfactometer (Galizia, Joerges, Küttner, Faber, \& Menzel, 1997). Odors used differed between animals and were 1-hexanol, 1-octanol, limonene and a mixture of limonene and linalool (all from Sigma-Aldrich). We excluded the 4 seconds before, during, and after stimulus presentation (a total of 12 seconds) from the analysis to ensure that no direct stimulus-response would contaminate the analysis. Great care was taken not to expose the animal to any test odor before the experiment, and no animal was investigated twice. Recordings were done using a Till-Photonics monochromator and CCD-based imaging system, Olympus BX50WI microscope, Olympus 20W dip objective, NA 0.5; dichroic 410, LP $440 \mathrm{~nm}$. Nine animals were examined. The experimental protocol complied with German law governing animal care.

2.2 Data Preanalysis. We calculated the ratio between the $340 \mathrm{~nm}$ and the $380 \mathrm{~nm}$ images for each measured point in time. In the FURA-dextran staining, individual glomeruli were clearly visible. We could thus identify the glomeruli on the basis of the morphological atlas of the honeybee AL (Galizia, Mcllwrath, \& Menzel, 1999). For each AL, we identified between 8 and 15 glomeruli (mean $=11.6 ; \mathrm{SD}=2.1$ ). Time traces for each glomerulus were calculated by spatially averaging the pixel values belonging to that glomerulus. For each AL, this procedure yielded two sets of matrices (before and after stimulation) consisting of between 8 and 15 glomeruli at 696 and 672 points in time, respectively ( 6 images per s for 2 minutes minus 4 and 8 seconds, respectively).

2.3 Mathematical Analysis. The measured traces were high-pass filtered (cut-off frequency $0.025 \mathrm{~Hz}$ ) to remove effects caused by a differential offset in different glomeruli, thus generating zero-mean time series. (Traces in Figures $1 \mathrm{~B}$ and $1 \mathrm{C}$ are not filtered). Odor responses were described as vectors whose components measure the maximum glomerular activity elicited by the specific odor. Glomeruli were categorized into activated or inhibited by an odor by visual inspection of the response trace. Pair-wise correlations were calculated as the correlation coefficient of their spontaneous activity. Lagged correlations were normalized to the correlation coefficient for zero 
lag and were calculated for relative shifts at 1 second intervals between -5 seconds and +5 seconds. All lags gave qualitatively the same results; Figure 3B shows the data for a lag of 3 seconds. The similarity between odor response and spontaneous activity was measured as their scalar product at every measured point in time. Correlation matrices across glomeruli were calculated for different time windows, taking the entire stretch before the stimulus, after the stimulus, or within four 1 minute intervals. We calculated the difference matrix as the difference between the correlation after and before stimulus. Significance for each element of the differential correlation matrix was calculated by bootstrapping the original data (2000 replications, $\alpha=0.05)$. A principal component analysis was performed on the correlation matrices and the difference matrix, and the first principal components (PCs) were extracted. The significances between glomerular activity patterns and the first PC of the spontaneous activity matrix were assessed by Kendall's non-parametric correlation coefficient $r$ (Press, Teukolsky, Vetterling, \& Flannery, 1992). Distributions of similarities across time and animals met normality conditions and were tested with an ANOVA.

\section{Results}

Odors evoke combinatorial patterns of activity across olfactory glomeruli in the first brain area that processes olfactory input, the insect antennal lobe (AL) or the vertebrate olfactory bulb (Hildebrand \& Shepherd, 1997; Korsching, 2002). These patterns can be visualized using optical imaging techniques (Galizia \& Menzel, 2001; Sachse \& Galizia, 2002). In honeybees, olfactory projection neurons (PNs) show a high degree of spontaneous activity in honeybees, that is, activity in the absence of an olfactory stimulus (Abel, Rybak, \& Menzel, 2001; Müller, Abel, Brandt, Zockler, \& Menzel, 2002), which can also be found in other species (Hansson \& Christensen, 1999). We have not investigated the driving force of this spontaneous background activity, but it appears likely to be controlled by a recurrent inhibitory network within the antennal lobe, since spontaneous activity is blocked by GABA and increased by the chloride channel blocker Picrotoxin, and may also rely on background activity from sensory neurons (Sachse \& Galizia, 2005). In many neurons, there is a direct relationship between the intracellular calcium concentration and the neuron's activity (Haag \& Borst, 2000; Ikegaya et al., 2004). PNs that show a high occurrence of action-potential bursts in electrophysiological recordings also show a continuous fluctuation of intracellular calcium levels at the same temporal scale (Galizia \& Kimmerle, 2004). Therefore, it is possible to use calcium imaging to study bursts of spontaneous activity in olfactory PNs (Sachse \& Galizia, 2002). To measure the spatiotemporal extent of such spontaneous activity patterns, we applied the calcium-sensitive dye FURA-dextran to the axon tract, leaving the AL, and obtained a backfill of PNs within the AL. Optical recording of the PNs confirmed that their intracellular calcium 
concentration was constantly changing; the spontaneous activity in the AL did not consist of longer periods of sustained activity but of short, sporadic activity bouts (see Figures 1A to 1D; see also additional data on the Web). These spontaneous events were glomerular in the sense that the spatial extent of individual activity spots always corresponded to a glomerulus in the morphological view. Their amplitude varied across both glomeruli and in time.

Stimulating the antennae with an odor led to characteristic odor-evoked activity patterns. The glomeruli activated in these patterns corresponded to those that were expected for the tested odors from previous studies (Galizia, Sachse, Rappert, \& Menzel, 1999; Sachse \& Galizia, 2002). The magnitude of intracellular calcium increases in an odor response was up to an order of magnitude higher than those of typical spontaneous activity fluctuations (see Figure 1B). After stimulation, odor-evoked calcium levels decayed back to baseline within a few seconds. This is consistent with measurements from olfactory receptor neurons, which in most cases stop firing at the end of an olfactory stimulus (de Bruyne, Foster, \& Carlson, 2001; Getz \& Akers, 1994), and supports the notion that the phenomena reported below are intrinsic to the AL and do not reflect an odor-specific structure in the input from receptors. This finding suggests that if there is a sensory memory within the AL, it cannot rely on persistent activity, that is, increased mean discharge rates after stimulation, as observed in other systems (Amit \& Mongillo, 2003; Fuster \& Alexander, 1971).

Figure 1: Unrewarded odor stimuli affect the spontaneous activity in the honeybee antennal lobe (AL), as illustrated by data from one bee. (A, left) Fluorescent image of the AL with projection neurons (PNs) stained with Rhodamin-dextran. (A, right) Sequence of spontaneous activity frames before, during (red frame), and after odor exposure (odor: 1-octanol). Note the similarity of the activity pattern at 80 seconds and 110 seconds with the odor response. Activity is color coded with equal scaling in all images. (For the entire measurement, go to http://galizia.ucr.edu/spontActivity.) (B) Raw traces for three identified glomeruli (red: T1-17; green: T1-33; black: T1-62) over a 240 second stretch. Stimulus lasted for 4 seconds, starting at $t=0$, as indicated by the bar. After the stimulus, activity fluctuations repeatedly co-occur. (C) Close-up view of two stretches in B. Open triangles indicate some occurrences where either glomerulus 17 (red) or 33 (green) were independently active. Closed triangles are those where both were active at the same time. Not all such instances are marked. (D) Projection of the spontaneous activity across all identified glomeruli before, during, and after odor presentation onto the activity during odor presentation itself. Filled triangles above the trace indicate all instances where the similarity measure is greater than 0.75 (dotted line). Such events are more prevalent after stimulus presentation, where the trace fluctuates more strongly rather than staying for longer periods in the high-similarity regime. The activity trace was high-pass filtered. 

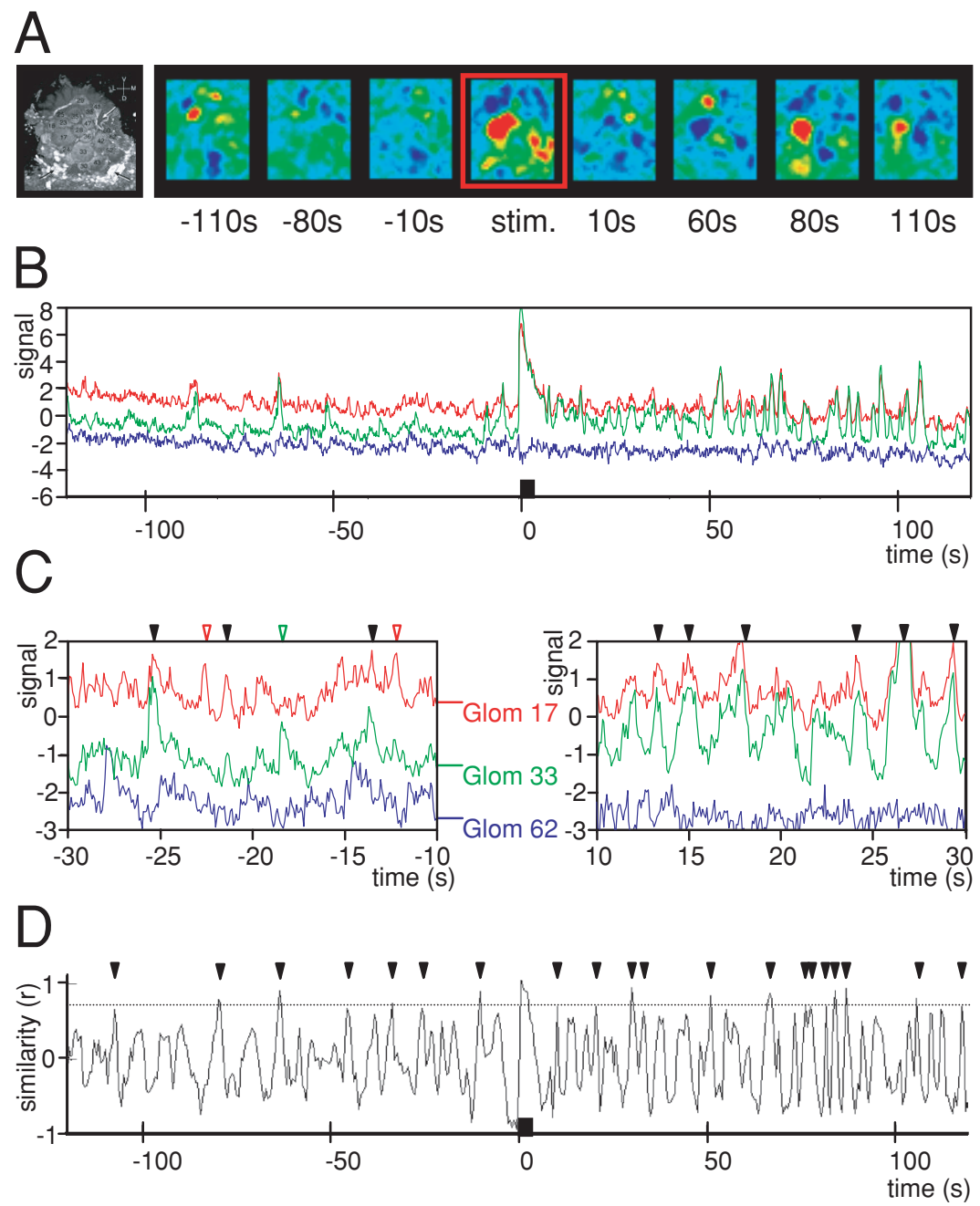

However, the relative timing of activity events in some glomeruli appeared to be altered. For instance, in Figure 1B, simultaneous fluctuations of glomerulus 17 and 33 are seen more frequently after the odor stimulus than before. We found that the glomeruli that increased their coactivity were those that had been activated by the odor stimulus. As shown in Figure 1C, coactive events between such glomeruli could also occur before stimulation (see filled triangles) and as independent activity bouts (empty triangles). After stimulation, coactive events were more frequent (filled triangles), and independent bouts were rare (none in the example here). Sensory memory in the AL may thus change the relative timing of spontaneous activity across 
AL glomeruli. To extend this to entire across-glomeruli patterns, we determined the similarity (Kendall's correlation) between odor response and spontaneous activity at every measured point in time (see Figure 1D). The resulting trace varies between 1 (perfect match, as at stimulus onset) and -1 . Because the activity traces were high-pass filtered before the projection, the values right before stimulus onset lead to the projection having value -1 . Instances where the similarity exceeds a threshold of 0.75 are marked by filled triangles. Clearly, after stimulation, spontaneous events resembling the odor-evoked response pattern became more prevalent: the spontaneous activity is on average more strongly attracted to the odor-evoked pattern.

We then investigated which specific properties of the spontaneous activity changed after stimulation. While overall activity increased in some individuals and decreased in others, across animals the standard deviation of the spontaneous activity was not affected by odor exposure ( $p=0.426$, Wilcoxon paired sample; see Figure 2A). This implies that the overall amplitude of the fluctuations did not increase; across animals, there was no increase in baseline activity. However, the total (summed) duration of spontaneous events that mimic the odor-evoked pattern (i.e., exceeding the 0.75 correlation threshold; see also Figure 1C) clearly differed after odor exposure ( $p<0.05$, Wilcoxon paired sample) and increased more than twofold (see Figure 2B). There was no change in the amplitude of each frequency component of the spontaneous activity, as demonstrated by approximately equal power spectra before and after stimulation (see Figure 2C), and no change in overall spontaneous activity, as seen when comparing the histogram of activity occurrences before and after stimulation (see Figure 2D). Thus, the short-term odor memory reported here is encoded only in the correlated spontaneous fluctuations rather than in their amplitude or their mean value. Which glomeruli change their relative activity timing? In order to address this question, we sorted all glomeruli pairs into three categories based on their response properties to the presented odor: pairs where the tested odor led to increased intracellular calcium concentration in both glomeruli, pairs where one partner increased and the other decreased its calcium level, and pairs where at least one of the two did not respond to the odor. We then analyzed their correlation before and after odor exposure (see Figure 3A). We found that coactive pairs increased their correlation; pairs with opposing sign decreased their correlation, and nonactive pairs remained unchanged. Thus, the correlation changes followed a Hebbian rule: pairs of glomeruli where both were excited or inhibited by the stimulus increased their spontaneous coherence after stimulation; pairs of glomeruli where one was excited and the other inhibited decreased their correlation. To test whether an unspecific change in spontaneous activity might explain the changed correlations, we shifted the activity traces against each other after and before odor stimulus and recalculated the pair-wise correlations on these traces (see Figure 3B). After relative shifting, a correlation purely caused by increased 
A

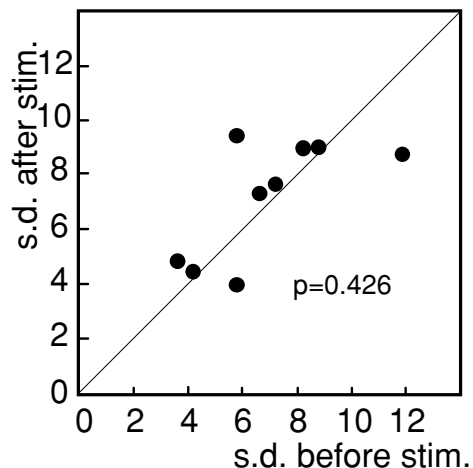

C

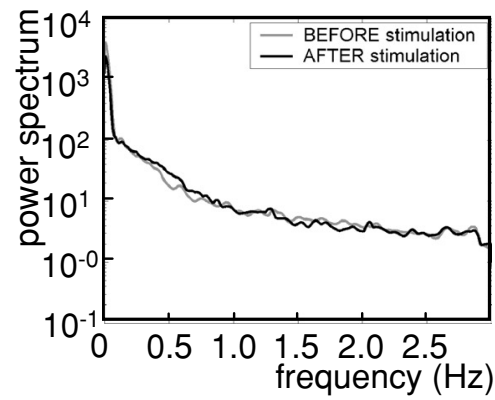

$\mathrm{B}$

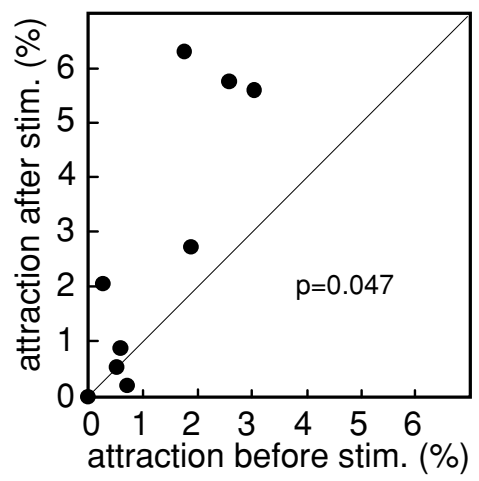

D

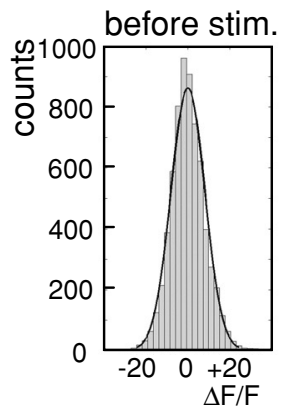

after stim.

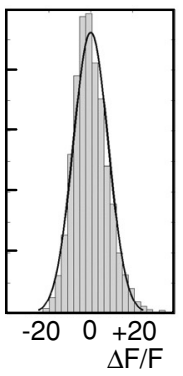

Figure 2: Statistical analysis of spontaneous AL activity before and after stimulation. (A) Standard deviations of the total AL activity fluctuations for each animal before odor exposure plotted against the same after odor exposure. No systematic change is observed ( $p=0.426$, Wilcoxon paired-sample test). (B) Odor-specific attraction, defined by the total (summed) duration of spontaneous events that closely resemble the odor-evoked activity pattern $(r>0.75$; see also Figure 1D), and given as percentage of the total recording time after odor exposure plotted against the same before odor exposure. After stimulation, attraction increased significantly across animals $(p<0.05$, Wilcoxon paired-sample test) and was on average more than twice as large as before stimulation. (C) Power spectrum of the spontaneous activity before (thin line) and after (thick line) odor stimulation, averaged over all glomeruli in all bees. (D) Histogram of spontaneous activity ( $\Delta \mathrm{F} / \mathrm{F}$ values in high-pass-filtered traces) before and after stimulation across glomeruli. The distribution does not change after the stimulus, indicating that there is no overall increase in spontaneous activity. Both distributions are slightly supergaussian (black lines represent fit to gaussian distributions). 

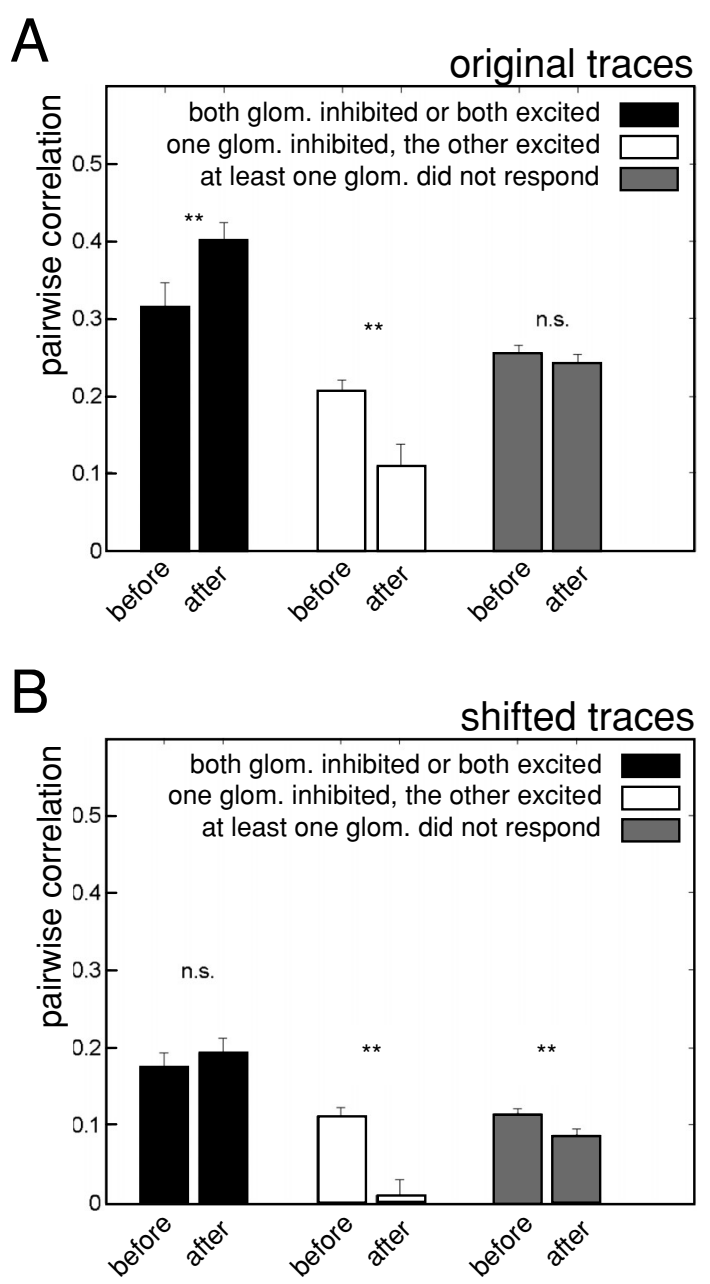

Figure 3: (A) Pair-wise correlation between olfactory glomeruli. As predicted by the Hebbian learning rule, pairs of glomeruli that were both excited or both inhibited by the odor significantly increased their correlation $(p \ll 0.05$, Wilcoxon paired-sample test); pairs of glomeruli where one was excited and the other one inhibited significantly decreased their correlation $(p \ll 0.05)$; pairs of glomeruli where at least one did not respond to the odor did not significantly change their correlation ( $p \gg 0.05$ ). (B) Pair-wise lagged correlation between olfactory glomeruli. By shifting the activity time series of each glomerulus with respect to each other (lag $=3$ seconds), the correlations between glomeruli were generally reduced, and the increased correlation of coactive glomeruli was abolished. The decrease of correlation in the other groups is a consequence of the statistical properties of these traces (see the text). A similar picture was obtained for any time lag larger than 1 second. 
activity should remain visible, while a correlation caused by specific timing of co-occurring events should decrease or disappear. We found that all correlation increases were lost or reduced, showing that the observed effect is due to a precisely timed coactivity rather than to an increased baseline activity. It should be noted that there is a small background correlation within the antennal lobe across all glomeruli, as evident in Figure 3A. Shifting the data reduces this background correlation (compare values between Figure 3A and Figure 3B); this effect also affects the traces after odor delivery, leading to the significances in Figure 3B for the non-co-excited glomeruli, where the decrease in correlation due to the stimulus and that due to shifting add up to a significant effect (see Figure 3B).

We next calculated the correlation matrix of glomerular activity, before and after stimulus presentation (left panels of Figures $4 \mathrm{~A}$ and $4 \mathrm{C}$ ) by calculating the pair-wise correlation between their activity time courses. We derived the correlation changes by subtracting the two matrices (left panel of Figure 4D). In the example shown, glomeruli 17, 28, 33, 36, and 52 increased their pair-wise correlation; they were coactive more often than before odor exposure. These glomeruli are those that responded most strongly to the odor (see Figure 4B). Pairs of glomeruli that were both inhibited during stimulation tended to increase their correlation, too, as shown by the pairs 23-49 and 29-37 in the left panel of Figure 4D. In contrast, most pairs where one glomerulus was excited by the odor and the other was inhibited decreased their correlation. This phenomenon is clearly apparent for pair $17-37,17-49$, or $23-52$.

Thus, it seems that the pair-wise correlation of glomerular activation patterns well after stimulus offset resembled the odor-evoked response patterns. To test this key hypothesis, we performed a principal component analysis (PCA). PCs are the eigenvectors of a correlation matrix. In particular, the first PC corresponds to the eigenvector whose eigenvalue has the largest magnitude. In our case, it represents the dominant pattern of spontaneous activity in the sense that its average projection onto the spontaneous activity is maximal (Beckerman, 1995). We performed a PCA of the correlation matrices before and after stimulation, as well as for the difference of both. We then quantified the correspondence between the first principal component (right panels in Figures 4A, $4 C$, and 4D) and the odor-evoked pattern (see Figure 4B, right side only). There was no significant relationship before odor stimulation $(r=0.31$, $p=0.143)$. After odor stimulation, the correspondence was highly significant $(r=0.79, p<0.001)$. The same holds for the first PC of the difference matrix $(r=0.74, p<0.001)$. This finding was confirmed across animals: the correlation matrices derived from the spontaneous activity after stimulation with an odor clearly reflected the pattern that was elicited by the odor, with mean correlation values between first PC and odor-evoked response of 0.52 after 1 minute and 0.39 after 2 minutes, as compared to 0.22 and 0.19 for 2 and 1 minutes before stimulation, respectively. These 
differences were highly significant (two-way ANOVA, $p<0.001$, with no significant difference between animals, $p=0.11$; see Figure $4 \mathrm{E}$ ).

In addition to this across-animal analysis, we asked (for each animal) whether the first PC corresponds to the odor response. One minute after stimulation, this was the case in six of nine animals; after 2 minutes, their number had dropped to three. This indicates that the memory trace encoded in the correlated activity fluctuations decreases on a timescale of a few minutes. As a control, we also tested the prestimulus condition and found no resemblance between the first PC and the odor response in any animal, as expected.

\section{Discussion}

As shown by our results, a single odor exposure without any predictive or associative value can lead to transient changes of the correlations between spontaneously active glomeruli that can last for more than 1 minute. Most notably, the pattern of activity that corresponds to the experienced odor is repeatedly "reactivated" during this period and thus constitutes a type of reverberation that is rather distinct from persistent activity (Amit \& Mongillo, 2003; Fuster \& Alexander, 1971). Hebb may have foreseen this

Figure 4: Spontaneous activity after odor stimulation represents an olfactory memory. Data in $A-D$ are from one animal. (A) Matrix of pair-wise correlations between glomerular activity before the stimulus is given (left). The diagonal elements of the matrix equal unity by definition and are depicted in black. Components of the first principal component (PC) of this matrix (right). The lack of significant correlation with the odor response is within the frame. (B) Glomerular activity pattern elicited by the odor (2-octanol). Glomeruli are arranged according to their activity strength. This sequence of glomeruli is kept throughout $A-D$. The left panel is deliberately left empty to ease comparison with the other panels of the figure. (C) As $A$, but after odor stimulation. The components of the first PC clearly differ from those before the stimulus $(A)$ and resemble the response pattern $(B)$. The significance of the correlation with the odor response is given within the frame. (D) As in $A$, but for the differences (after or before) of pair-wise correlations. Nonsignificant entries of the matrix (by bootstrap analysis; see the text) have been set to zero and are shown in white; the diagonal elements equal zero by definition and are depicted in gray. As in $C$ there is a statistically significant correlation to the odor response pattern $(B)$. (E) Population data. Box plot of Kendall's correlation between the first eigenvector and the odor response calculated from correlation matrices 2 minutes and 1 minute before and after stimulus delivery. There is a highly significant increase in the correlation. Numbers above the box plots indicate how many animals had a significant correlation between the first PC and the odor response. In agreement with our other results, this correlation was not significant for those bees for which the attraction did not increase after odor presentation (see Figure 2C). 

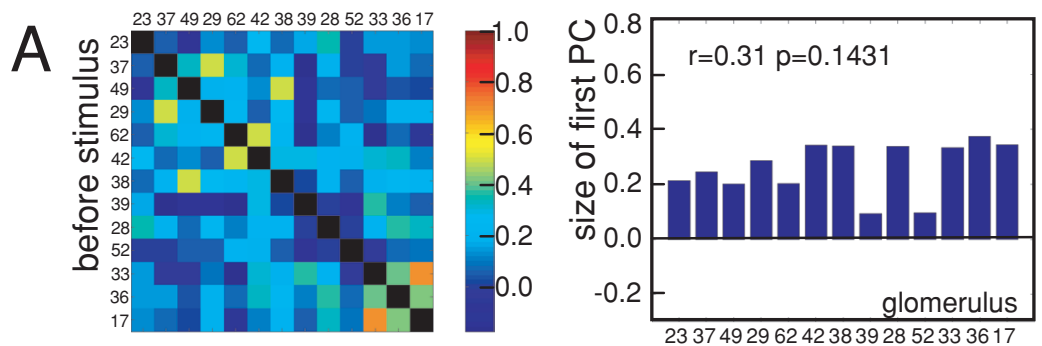

B
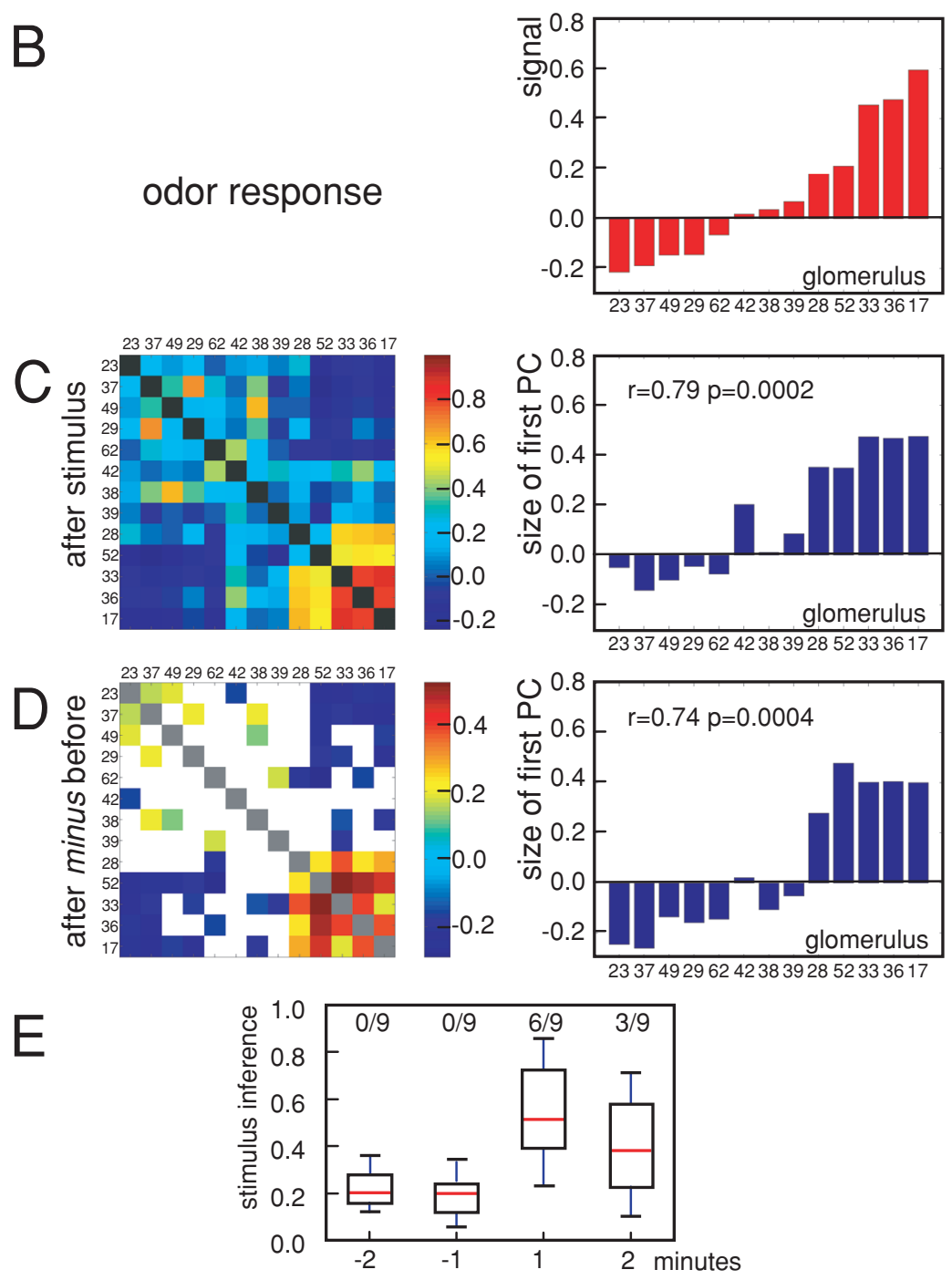
possibility when he discussed both "persistence or repetition of a reverberatory activity" (Hebb, 1949, p. 62).

Statistical properties of spontaneous activity patterns have been investigated in other systems, notably the mammalian cortex (Ikegaya et al., 2004). In this structure, the correlation between units is related to the behavioral state of the animal (Vaadia et al., 1995). A possible mechanism for creating specific firing patterns between neurons is provided by the concept of synfire-chains (Abeles, 1991; Durstewitz, Seamans, \& Sejnowski, 2000). In this study we have not investigated the relationship of individual spikes, but that of entire activity bursts, which are reflected in the calcium increases at the temporal resolution of our measurements. Therefore, a direct comparison should be approached with caution.

Which are the neurons that mediate the observed correlation changes? Since the changes occur between glomeruli, neurons connecting between glomeruli are likely candidates. In the honeybee, there are up to 4000 neurons local to the AL (Flanagan \& Mercer, 1989; Fonta, Sun, \& Masson, 1993; Galizia \& Menzel, 2000). These neurons are not a uniform group: they have different transmitters (GABA, histamine), different morphologies (heterogeneous, homogeneous), and innervate different groups of glomeruli. Further work should elucidate which subpopulation of LNs accounts for stimulus-specific modifications of the glomerular fluctuations. Clearly, however, the changes follow a Hebbian correlation rule: glomeruli that are coactivated during a stimulus probably increase a (putative) reciprocal excitatory connection and/or decrease an inhibitory connection, so that their co-occurrence in a spontaneous activity event becomes more likely than before stimulation (see Figure 3). However, since the network does not consist of pair-wise connections, this description is certainly too simplistic. More work is needed to identify the mechanisms that may account for these findings. The stimulus may induce short-term changes of synaptic or membrane properties, which are known to influence spontaneous activity patterns (Kenet, Bibitchkov, Tsodyks, Grinvald, \& Arieli, 2003; Tsodyks, Kenet, Grinvald, \& Arieli, 1999). Stimulus-dependent modifications may, however, also be purely dynamic in the sense of "a memory trace that is wholly a function of a pattern of neural activity, independent of any structural change" (Hebb, 1949).

The observed multiglomerular activity fluctuations are readily interpreted if we visualize the AL as a network with odor-specific attractors and a high level of spontaneous activity (Galán, Sachse, Galizia, \& Herz, 2004). If an odor is presented, the basin of attraction corresponding to this stimulus is increased and biases the network fluctuations toward the odorspecific pattern. This may lead to the network enhancing the representation of that odor relative to others, as seen in Figure 2C. Such a short-term memory effect has been observed in locusts (Stopfer \& Laurent, 1999). In that study, the coherence of PN activity increased when an odor stimulus was iterated. If the sensory memory of the previous (but same) stimulus was 
still active, it would cause a reduced threshold for that pattern and thus facilitate a more coherent response, that is, more strongly synchronized PN activity, as reported (Stopfer \& Laurent, 1999). In the rat olfactory bulb, exposure to an odor slightly modifies the response profile of mitral cells (Fletcher \& Wilson, 2003), a finding that might indicate that similar changes in the interglomerular neural network occur in mammals as those observed here.

It remains unclear, though, under what conditions, if any, changes are read out by other brain areas, that is, if spontaneous activity bouts can be coincident and cause spurious "remembrances" of the experienced odor, or whether these bouts are "perceived" as odor whiffs by the animal. By briefly changing the network activity, they might also play a role in classical conditioning of odors with appetitive rewards (Menzel \& Müller, 1996). Let us also note that even if an external observer can retrieve the odor from the spontaneous activity, this does not prove that the animal actually uses this information. Nevertheless, the observed correlation changes are a robust and predictable phenomenon that occurs in the AL; by itself, this unexpected finding invites further investigation.

In conclusion, we have revealed traces of sensory memory, in vivo, and have demonstrated that a single odor stimulus can modify the spontaneous activity of olfactory glomeruli. As traditional paradigms investigating Hebbian reverberations have exclusively focused on persistent activity after stimulation, not on correlated activity fluctuations, it is to be expected that future investigations along the lines of our study may reveal previously overlooked memory traces in many other neural systems.

\section{Acknowledgments}

The work of M. W. and R. M. was supported by the Deutsche Forschungsgemeinschaft (SFB 515).

\section{References}

Abel, R., Rybak, J., \& Menzel, R. (2001). Structure and response patterns of olfactory interneurons in the honeybee, Apis mellifera. J. Comp. Neurol., 437(3), 363-383.

Abeles, M. (1991). Corticonics: Neural circuits of the cerebral cortex. Cambridge: Cambridge University Press.

Amit, D. J., \& Mongillo, G. (2003). Selective delay activity in the cortex: Phenomena and interpretation. Cereb. Cortex, 13(11), 1139-1150.

Beckerman, M. (1995). Adaptive cooperative systems. New York: Wiley.

Clark, R. E., Manns, J. R., \& Squire, L. R. (2002). Classical conditioning, awareness, and brain systems. Trends. Cogn. Sci., 6(12), 524-531.

Crowder, R. G. (2003). Sensory memory. In J. H. Byrne (Ed.), Learning and memory (2nd ed., pp. 607-609). New York: Macmillan. 
de Bruyne, M., Foster, K., \& Carlson, J. R. (2001). Odor coding in the Drosophila antenna. Neuron, 30(2), 537-552.

Del Giudice, P., Fusi, S., \& Mattia, M. (2003). Modelling the formation of working memory with networks of integrate-and-fire neurons connected by plastic synapses. J. Physiol. Paris, 97(4-6), 659-681.

Durstewitz, D., Seamans, J. K., \& Sejnowski, T. J. (2000). Neurocomputational models of working memory. Nat. Neurosci., 3 Suppl., 1184-1191.

Flanagan, D., \& Mercer, A. R. (1989). Morphology and response characteristics of neurones in the deutocerebrum of the brain in the honeybee Apis mellifera. J. Comp. Physiol. (A), 164, 483-494.

Fletcher, M. L., \& Wilson, D. A. (2003). Olfactory bulb mitral-tufted cell plasticity: Odorant-specific tuning reflects previous odorant exposure. J. Neurosci., 23(17), 6946-6955.

Fonta, C., Sun, X. J., \& Masson, C. (1993). Morphology and spatial distribution of bee antennal lobe interneurones responsive to odours. Chemical Senses, 18, 101-119.

Fuster, J. M., \& Alexander, G. E. (1971). Neuron activity related to short-term memory. Science, 173(997), 652-654.

Galán, R. F., Sachse, S., Galizia, C. G., \& Herz, A. V. (2004). Odor-driven attractor dynamics in the antennal lobe allow for simple and rapid olfactory pattern classification. Neural Comput., 16(5), 999-1012.

Galizia, C. G., Joerges, J., Küttner, A., Faber, T., \& Menzel, R. (1997). A semi-in-vivo preparation for optical recording of the insect brain. J. Neurosci. Methods, 76(1), 61-69.

Galizia, C. G., \& Kimmerle, B. (2004). Physiological and morphological characterization of honeybee olfactory neurons combining electrophysiology, calcium imaging and confocal microscopy. J. Comp. Physiol. A, 190(1), 21-38.

Galizia, C. G., Mcllwrath, S. L., \& Menzel, R. (1999). A digital three-dimensional atlas of the honeybee antennal lobe based on optical sections acquired by confocal microscopy. Cell Tissue Res., 295(3), 383-394.

Galizia, C. G., \& Menzel, R. (2000). Odour perception in honeybees: Coding information in glomerular patterns. Curr. Opin. Neurobiol., 10(4), 504-510.

Galizia, C. G., \& Menzel, R. (2001). The role of glomeruli in the neural representation of odours: Results from optical recording studies. J. Insect. Physiol., 47(2), 115130.

Galizia, C. G., Sachse, S., Rappert, A., \& Menzel, R. (1999). The glomerular code for odor representation is species specific in the honeybee Apis mellifera. Nat. Neurosci., 2(5), 473-478.

Getz, W. M., \& Akers, R. P. (1994). Honeybee olfactory sensilla behave as integrated processing units. Behav. Neural. Biol., 61(2), 191-195.

Giurfa, M., Zhang, S., Jenett, A., Menzel, R., \& Srinivasan, M. V. (2001). The concepts of "sameness" and "difference" in an insect. Nature, 410(6831), 930-933.

Grossmann, K. E. (1971). Belohnungsverzögerung beim Erlernen einer Farbe an einer künstlichen Futterstelle durch Honigbienen. Z. Tierpsychol., 29, 28-41.

Haag, J., \& Borst, A. (2000). Spatial distribution and characteristics of voltage-gated calcium signals within visual interneurons. J. Neurophysiol., 83(2), 1039-1051.

Hansson, B. S., \& Christensen, T. A. (1999). Functional characteristics of the antennal lobe. In B. S. Hansson (Ed.), Insect olfaction (pp. 125-161). Heidelberg: Springer. 
Hebb, D. O. (1949). The organization of behavior: A neuropsychological theory. New York: Wiley.

Hildebrand, J. G., \& Shepherd, G. M. (1997). Mechanisms of olfactory discrimination: Converging evidence for common principles across phyla. Annu. Rev. Neurosci., 20,595-631.

Ikegaya, Y., Aaron, G., Cossart, R., Aronov, D., Lampl, I., Ferster, D., \& Yuste, R. (2004). Synfire chains and cortical songs: Temporal modules of cortical activity. Science, 304(5670), 559-564.

Kenet, T., Bibitchkov, D., Tsodyks, M., Grinvald, A., \& Arieli, A. (2003). Spontaneously emerging cortical representations of visual attributes. Nature, 425(6961), 954-956.

Korsching, S. (2002). Olfactory maps and odor images. Curr. Opin. Neurobiol., 12(4), 387-392.

Menzel, R. (2001). Searching for the memory trace in a mini-brain, the honeybee. Learn. Mem., 8(2), 53-62.

Menzel, R., \& Müller, U. (1996). Learning and memory in honeybees: From behavior to neural substrates. Annu. Rev. Neurosci., 19, 379-404.

Müller, D., Abel, R., Brandt, R., Zockler, M., \& Menzel, R. (2002). Differential parallel processing of olfactory information in the honeybee, Apis mellifera L. J. Comp. Physiol. A., 188(5), 359-370.

Press, W. H., Teukolsky, S. A., Vetterling, W. T., \& Flannery, B. P. (1992). Numerical recipes in C: The art of scientific computing. Cambridge: Cambridge University Press.

Sachse, S., \& Galizia, C. G. (2002). Role of inhibition for temporal and spatial odor representation in olfactory output neurons: A calcium imaging study. J. Neurophysiol., 87(2), 1106-1117.

Sachse, S., \& Galizia, C. G. (2005). Topography and dynamics of the olfactory system. In S. Grillner (Ed.), Microcircuits: The interface between neurons and global brain function. Cambridge, MA: MIT Press.

Sejnowski, T. J. (1977). Storing covariance with nonlinearly interacting neurons. J. Math. Biol., 4(4), 303-321.

Stopfer, M., \& Laurent, G. (1999). Short-term memory in olfactory network dynamics. Nature, 402(6762), 664-668.

Tsodyks, M., Kenet, T., Grinvald, A., \& Arieli, A. (1999). Linking spontaneous activity of single cortical neurons and the underlying functional architecture. Science, 286(5446), 1943-1946.

Vaadia, E., Haalman, I., Abeles, M., Bergman, H., Prut, Y., Slovin, H., \& Aertsen, A. (1995). Dynamics of neuronal interactions in monkey cortex in relation to behavioural events. Nature, 373(6514), 515-518. 